\title{
Surface Plasmon Polariton Excitation in Metallic Layer Via Surface Relief Gratings in Photoactive Polymer Studied by the Finite-Difference Time-Domain Method
}

\author{
Pawel Karpinski • Andrzej Miniewicz
}

Received: 3 February 2011 / Accepted: 2 May 2011 / Published online: 11 May 2011

(C) The Author(s) 2011. This article is published with open access at Springerlink.com

\begin{abstract}
We performed numerical investigations of surface plasmon excitation and propagation in structures made of a photochromic polymer layer deposited over a metal surface using the finite-difference time-domain method. We investigated the process of light coupling into surface plasmon polariton excitation using surface relief gratings formed on the top of a polymer layer and compared it with the coupling via rectangular ridges grating made directly in the metal layer. We also performed preliminary studies on the influence of refractive index change of photochromic polymer on surface plasmon polariton propagation conditions.
\end{abstract}

Keywords Plasmonics · Localized surface plasmons · Photochromic polymers $\cdot$ Surface relief gratings

\section{Introduction}

Quasiparticles that can propagate at the interface between conductor and dielectric due to collective charge oscillations are known as surface plasmon polaritons (SPP). Various forms of them have been recognized ranging from freely propagating electron density waves along metal surfaces to localized electron oscillations on metal nanoparticles. Unique properties of surface plasmons, i.e., their sensitivity to a changing environment at a nanoscale, enable a wide range of practical applications, including light

\section{P. Karpinski $(\bowtie) \cdot$ A. Miniewicz}

Institute of Physical and Theoretical Chemistry,

Wroclaw University of Technology,

Wroclaw, Poland

e-mail: pawel.karpinski@pwr.wroc.pl guiding [1], manipulation at nanoscale [2], biodetection $[3,4]$, high-resolution optical imaging [5], optical switching $[6,7]$, and many others [8].

We limit ourselves to plasmon excitation on metallic surface in contact with photochromic polymer. There are many ways to excite the SPP on a metal surface [9]: using prism coupling in Otto or Kretschmann configurations, near-optical field excitation, highly focused optical beam technique, excitation on discontinuities, and imperfections in metallic layers like holes, ridges, or their arrays. In this work, we focused our attention to light coupling into SPP occurring on a periodic array of corrugated polymer surface and rectangular metallic ridges that could be fabricated on a metal surface [10]. Light diffracted on periodic structures can fulfill necessary conditions of momentum matching with SPP and increase the plasmon excitation efficiency. Grating excitation method limits the dispersion distribution of excited SPP to a very narrow frequency range being precisely defined by the grating parameters and metal dielectric permittivity. Generally, grating structures offer a possibility of tuning frequency of the excited SPP by changing grating geometrical parameters, like grating period, ridge height or grating duty factor [11], and properties of dielectric medium. Recent studies [12] have shown that an efficiency of SPP excitation by grating method can reach $\sim 22 \%$, what usually is sufficient for experimental observations of plasmons as well as for fabrication of plasmonic sensors [13] and light switching devices [8]. In this work, we investigate the possibility of SPP excitation on finite metal and polymeric gratings and plasmon frequency shift caused by an external stimulus in the form of light which changes the polymer refractive index. 


\section{Excitation of SPP and FDTD Method Description}

Light coupling on the grating and subsequent excitation of the SPP requires energy and momentum conservation of the photon and surface plasmon polariton:

$\hbar \omega_{\text {light }}=\hbar \omega_{\mathrm{SPP}}$,

$k \cdot \sin (\theta)+m K= \pm \beta$,

where $k=2 \pi / \lambda$ is a propagation vector of light, $\beta$ is an SPP propagation vector, $K=2 \pi / \Lambda$ is a wavevector of an infinite grating and $m$ is an integer number. The same conditions must be fulfilled when the SPP is excited in a flat metallic layer due to light diffraction on a surface relief grating (SRG) formed in a thin polymeric layer deposited over the metal surface. The surface relief grating on polymer layer can be made by a holographic method, and in that case, its shape could be described by a cosine function.

In fact, we performed calculations for a few different shapes of ridges and found that differences are not significant, therefore in this article we limit our study only to sinusoidal shapes of the grating that can be easily prepared by holographic laser illumination. In Fig. 1, we schematically show the rectangular metallic grating over bulk metal together with the chosen coordinate system and grating $(K)$, light $(k)$, and SPP $\left(\beta_{\mathrm{SPP}}\right)$ wavevectors. The surface relief grating formed in a polymeric layer covering the flat metal surface is also shown in Fig. 1. It is wellknown [8] that for a flat metal-dielectric interface, an SPP wave vector $\beta$ fulfills a dispersion equation:

$\beta(\omega)=\sqrt{\frac{\varepsilon_{\mathrm{d}} \cdot \varepsilon_{\mathrm{m}}(\omega)}{\varepsilon_{\mathrm{d}}+\varepsilon_{\mathrm{m}}(\omega)}}$,

where $\varepsilon_{\mathrm{d}}$ is a dielectric permittivity of a polymer or air assumed as nondispersive ones in the respective frequency range and $\varepsilon_{\mathrm{m}}(\omega)$ is a complex permittivity of a metal which can be described within the Drude-Lorentz oscillator approximation [8] as:

$\varepsilon_{\mathrm{m}}(\omega)=\varepsilon_{\infty}-\frac{\omega_{p}^{2}}{\omega^{2}+i \gamma \omega}+\sum_{j} \frac{f_{j} \omega_{j}^{2}}{\omega_{j}^{2}-\omega_{j}^{2}-i \gamma_{j} \omega}$,

Fig. 1 Schematic view of metal grating and surface relief grating in polymer over a flat metal. Momentum conservation conditions of light coupling into SPP on metal grating with left $\left(\beta_{\mathrm{LSPP}}\right)$ and right $\left(\beta_{\mathrm{RSPP}}\right) \mathrm{SPP}$ propagation constants are given as well
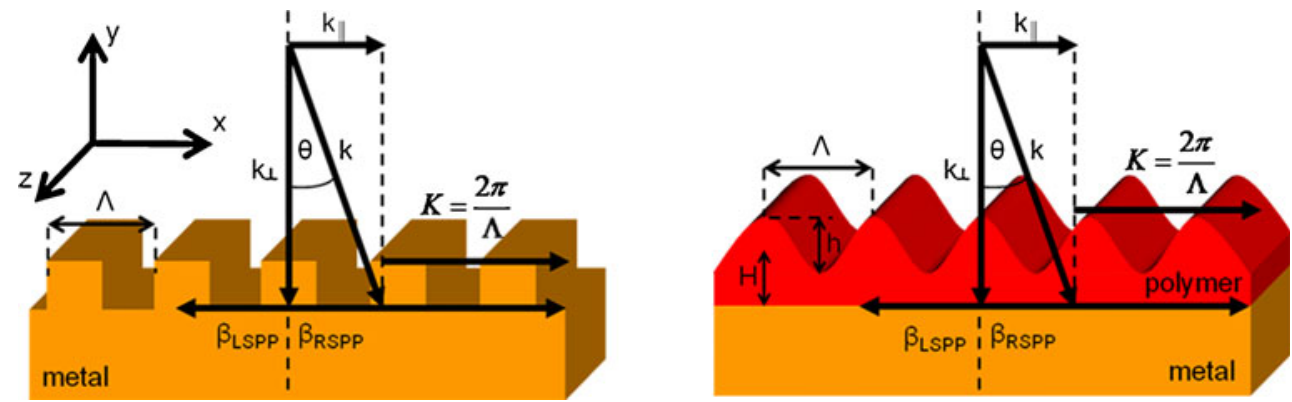

where $\omega_{\mathrm{p}}=\sqrt{\frac{\mathrm{Ne}^{2}}{\varepsilon_{0} m_{\mathrm{e}}}}$ is the plasma frequency and $\gamma$ is the dumping constant of a free electron gas, $f_{j}, \omega_{j}, \gamma_{j}$ are strength, frequency, and dumping constants of the $j$ th Lorentz's oscillator. We use the two-dimensional (2D) finite-difference time-domain (FDTD) method with auxiliary differential equation for Lorentz-like media having permittivity in the form given by an equation (Eq. 4). The FDTD method relies on the numerical solving of the Maxwell equations for electromagnetic wave propagation on the so-called Yee grid based on the second-order central differences [14]. The FDTD method is successfully used in simulation of plasmonic and optical switching structures [15-17].

In order to model the dielectric permittivity for pure gold $\mathrm{Au}$ ) layer we used a dielectric permittivity at infinite frequencies, plasma frequency, and damping coefficient as given in Ref. [18, 19], i.e., $\varepsilon_{\infty}=6.2136, \omega_{\mathrm{p}}=1.3323 \times 10^{16}$ $\mathrm{Hz}$, and $\gamma=1.3235 \times 10^{14} \mathrm{~Hz}$. For the sake of simplicity (to shorten calculation time) we limited metal permittivity just to the Drude model, i.e., neglecting all Lorentz's terms in Eq. (4). We also assumed that the polymer layer is dispersionless and lossless, so its dielectric permittivity is constant and real, therefore for optical frequencies we took: $\varepsilon_{\mathrm{d}}=n_{\mathrm{d}}^{2}$ where $n_{\mathrm{d}}$ is a refractive index of the polymer. The period of the Yee grid is $d x=d y=5 \mathrm{~nm}$ and the time period, $\mathrm{dt}=10^{-17} \mathrm{~s}$. The light source is taken as a sum of linear monochromatic sources with wavelengths ranging from 300 to $1,000 \mathrm{~nm}$ every $10 \mathrm{~nm}$ which are what defines a discrete polychromatic light source. Incoming light polarization is TM with a magnetic field component parallel to the $z$-axis. The electromagnetic wave was calculated either using Bloch boundary conditions for grating infinitely in the $x$ direction or without it for finite gratings having several ridges along the $x$-axis. The structure is surrounded by the properly chosen boundary conditions represented here by Berenger's perfect matching layers for uniaxial mediums with refraction factor $R=10^{-4}$ [14]. A light beam has been assumed to have a Gaussian intensity profile in calculations of finite grating structures and a flat profile in calculations of infinite gratings.

An example of SPP excitation in a finite grating structure with EM field components calculated by the FDTD method is shown in Fig. 2. The FDTD method gives
The 2D structure is covered with the uniform Yee grid. 
a time dependence of the electric and magnetic field components within the structure. In order to obtain results in the function of wavelength, the Fourier transformation of the electric or magnetic fields were calculated: $\langle H(\lambda)\rangle=$ $F\{H(t)\}$ for the magnetic field or $\left\langle E_{\perp}(\lambda)\right\rangle=F\left\{E_{\perp}(t)\right\}$ for the electric field perpendicular to the metal surface, where $\lambda=2 \pi c / \omega$ is the light wavelength in the vacuum.

\section{Results of Simulation of the SPP Excitation on Different Gratings}

The SPP resonance peak spectrum for a purely metallic (Au) grating formed by rectangular protrusion ridges of $\Lambda=$ $700 \mathrm{~nm}$ period is shown in Fig. 3. We calculated a magnetic field strength in a small distance from the metal surface and light source position which illuminated the sample at an incidence angle $\theta=15^{\circ}$. The three main peaks correspond to momentum conservation laws as shown in Fig. 3. The central peak corresponds to the forward SPP propagation with respect to the position of the light source while the remaining two to the back SPP propagation. The absorption of incident photon energy in metal makes the amplitudes of the respective plasmons differ significantly depending on their wavelengths $\lambda_{\mathrm{SPP}}$. Plasmons could be excited in a flat metal layer also due to light diffraction on a surface relief grating made on the top of the photochromic polymer film. It was interesting to compare, for the same light source, the efficiency of SPP excitation on metal surface due to metal ridges and due to surface relief of the same period inscribed in the top of the polymer layer. The dielectric SRG has been assumed to have a sinusoidal shape because such a shape can easily be obtained in azo-substituted polymers by laser holographic method [20]. In all simulations, the refractive index of the photochromic polymer is assumed to be $n=1.5$. Calculating the SPP excitation efficiency on metal grating with a rectangular shape, we kept the same height of the ridges $h$ as was the amplitude of a relief grating. Preliminary calculations of the electric field distribution in metal-polymer-air (MPA) structure resulted in the obser-

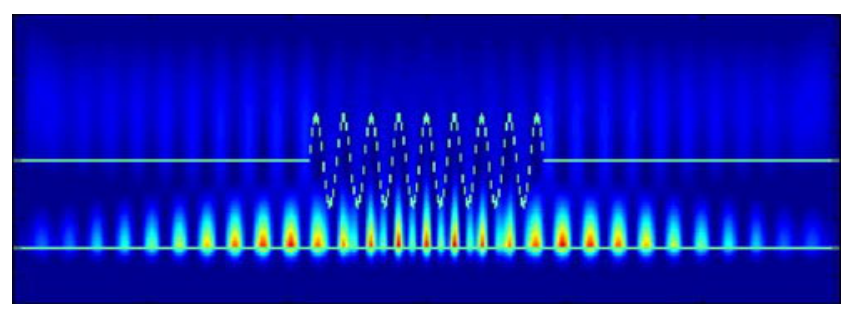

Fig. 2 Plot of calculated electromagnetic field intensity of normally incident light with wavelength in vacuum $\lambda=600 \mathrm{~nm}$ coupled into the SPP on the dielectric layer $(n=1.5)$ with surface relief grating and a flat metal surface underneath. Parameters of SRG are $H=100 \mathrm{~nm}, h=$ $100 \mathrm{~nm}$, and $\Lambda=500 \mathrm{~nm}$ (cf. Fig. 1)

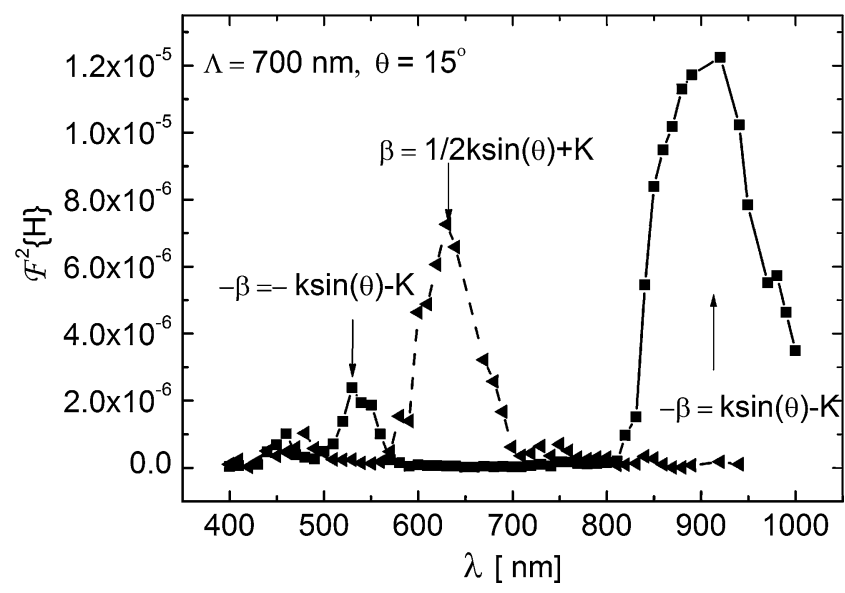

Fig. 3 Plot calculated by FDTD method. SPP resonance bands for metal grating with a period of $\Lambda=700 \mathrm{~nm}$ and at light incidence angle $\theta=15^{\circ}$. For each band, a corresponding momentum conservation relation is indicated

vation of several resonances (cf. Fig. 4). Their appearance is connected with light wave guiding properties of the polymer layer itself. Therefore, it was necessary to distinguish between purely electromagnetic wave-guided p-polarized modes (dielectric modes) in polymer and true SPP modes characteristic for the metal-dielectric interface. To do this, we precisely calculated the light energy distribution in the bulk of metal, polymer, and air after the illumination of the structure with polychromatic light. The structures were made of infinite thickness metal with a polymer layer over it (having a mean thickness $H$ from 100 to $1,000 \mathrm{~nm}$ and refractive index $n_{\mathrm{d}}=1.5$ ). Additionally, the polymer layer had an infinite surface relief grating of period $\Lambda=500 \mathrm{~nm}$ with a relief amplitude of $h=100 \mathrm{~nm}$. For comparative purposes, we calculated similar wave guiding structures with air instead of a metal, i.e., classic airpolymer layer-air (APA) planar waveguide. For structures with relatively thick polymer layers $H$ we have found a series of well-defined modes (c.f. Fig. 4c) propagating at various frequencies or wavelengths. Four among the modes appeared both in the MPA and APA structures, what is demonstrated in Fig. 4a, b. The profiles of field distribution are characteristic for the lowest and subsequent higher planar waveguide modes. We concluded that these modes are not the SPP modes, but dielectric ones [21] and labeled them from $\mathrm{m} 0$ to $\mathrm{m} 3$. Their characteristic wavelengths evolved together with polymer thickness $H$ (cf. Fig. 4c). Exclusively for MPA structures, two additional modes appear, which we consider as SPP ones: for $\lambda=330 \mathrm{~nm}$ and for $\lambda=690-790 \mathrm{~nm}$ (cf. Fig. $4 \mathrm{~d}$ inset). The former SPP $330 \mathrm{~nm}$ mode is highly confined in metal with very short characteristic propagation distance $L=(2 \operatorname{Im}(\beta))^{-1}$ equal to $0.58 \mu \mathrm{m}$. The latter SPP mode is much less confined in the metal, and its propagation distance is considerably larger, $L=11.66 \mu \mathrm{m}$. Both plasmonic modes have maximum of 
a

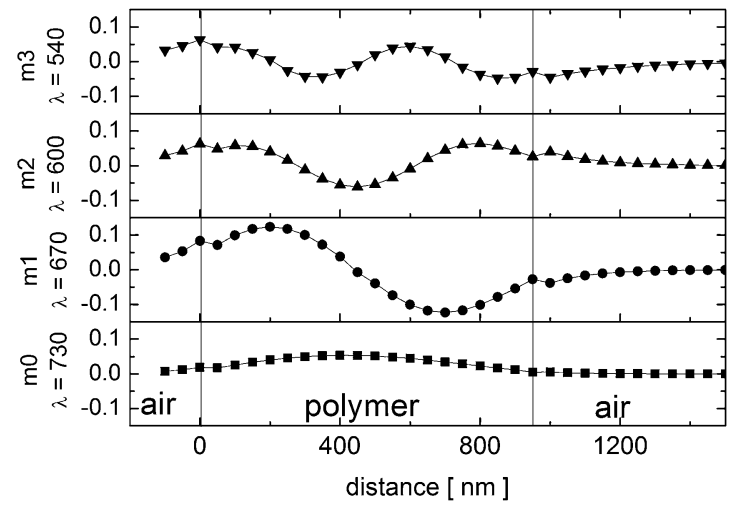

b

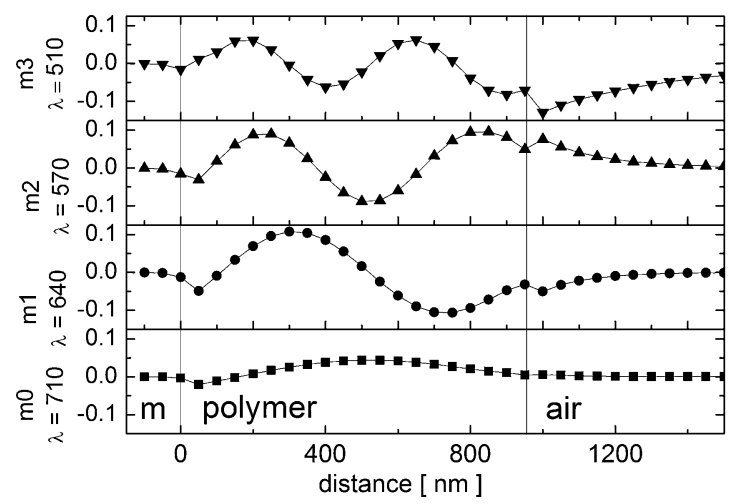

Fig. 4 Calculated by FDTD method. Profiles of electric field distribution in air-polymer-air (a) and metal-polymer-air (b) structures with $H=950 \mathrm{~nm}$. Modes labeled $m 0, m 1, m 2$, and $m 3$ are the light-guided modes in the polymer layer. Dependence of the mode

electric field at the metal-dielectric interface and both decay exponentially in the direction $y$ perpendicular to the metal and polymer interface. An electric field amplitude for the SPP at $790 \mathrm{~nm}$ in the vicinity of the metal-polymer interface in the function of distance $y$ from the interface is

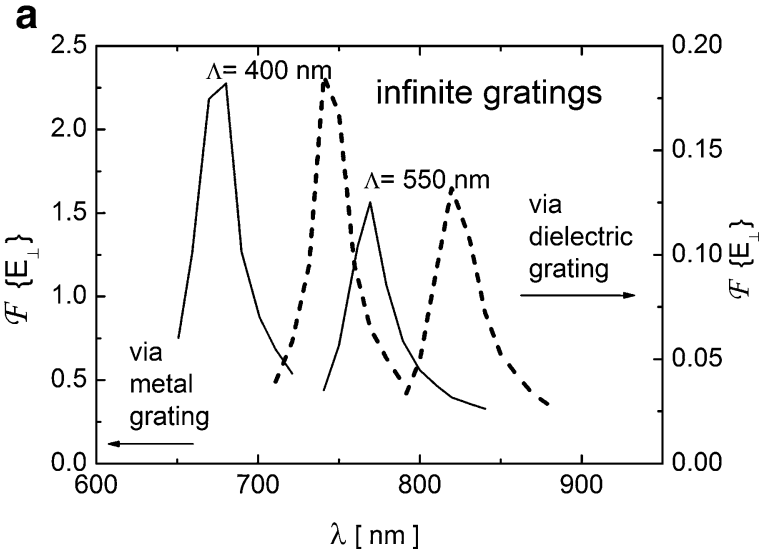

Fig. 5 Plot of electric field amplitude perpendicular to the metal surface $F\left\{E_{\perp}\right\}$ inside the infinite grating. Comparison of electric field amplitudes for two exemplary grating periods $\Lambda=400 \mathrm{~nm}$ and $\Lambda=$

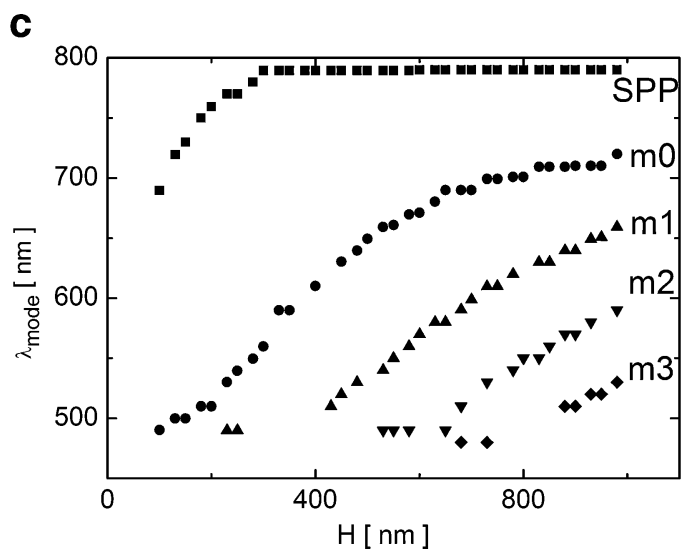

d

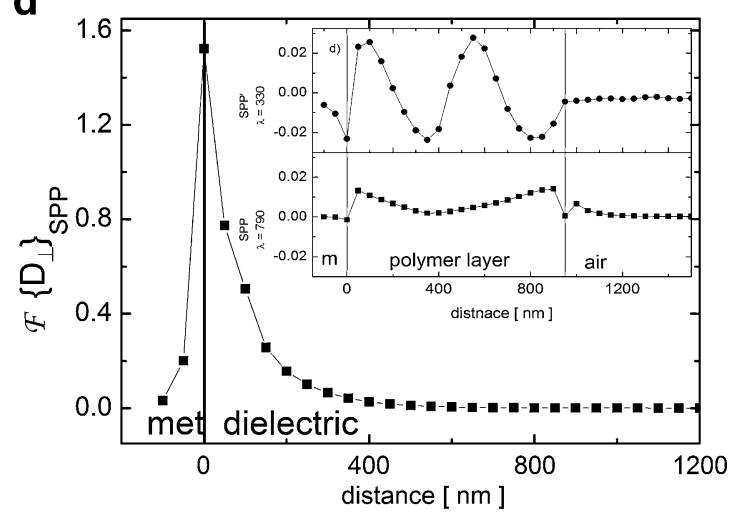

frequency on the polymer layer thickness, $H(\mathbf{c})$. The electric field distribution close to the gold-polymer interface for SPP at $790 \mathrm{~nm}$; inset: electric field distribution profiles for two SPP modes (d)

shown in Fig. 4d; the field penetration depths are $d_{\mathrm{Au}}=$ $50 \mathrm{~nm}$ and $d_{\text {polym }}=200 \mathrm{~nm}$, respectively. In the forthcoming calculations presented in this paper, we will investigate the properties of the SPP mode characterized by a larger propagation length, $L$.

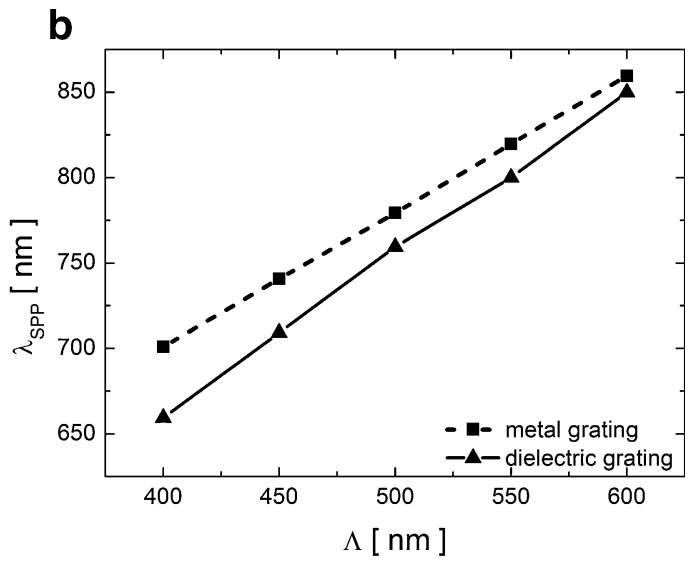

$550 \mathrm{~nm}$ (a) and dependence of excited SPP wavelength on period of the grating $\Lambda$ (b) for metallic and dielectric gratings 


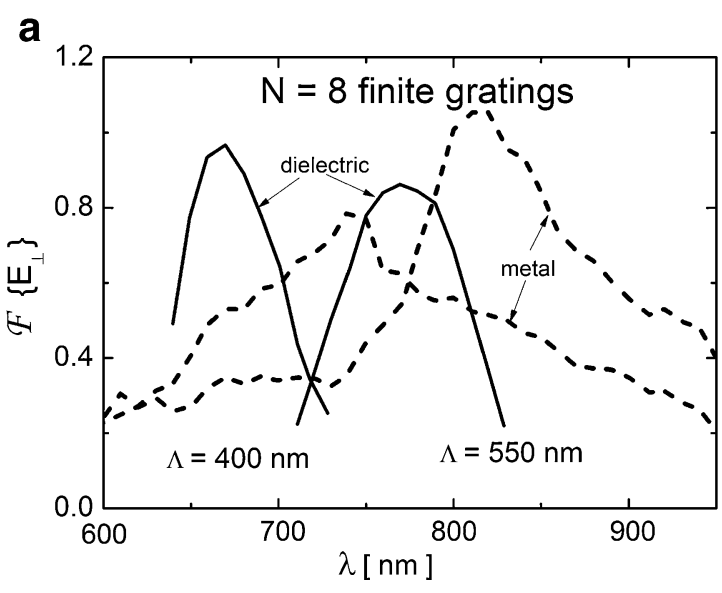

Fig. 6 Plot of electric field amplitude perpendicular to the metal surface $F\left\{E_{\perp}\right\}$ for the finite grating at a constant distance from the grating end. Comparison of electric field amplitudes for two exemplary grating periods $\Lambda=400 \mathrm{~nm}$ and $\Lambda=550 \mathrm{~nm}$ (a) and

In order to investigate the properties of SPP excited via SRG grating inscribed on a polymer surface and directly on a metal grating, we performed numerical investigations for the case of finite gratings having only $N=8$ ridges and infinite gratings to which the Bloch boundary conditions were applied. Calculations were performed for two exemplary grating periods $\Lambda=400 \mathrm{~nm}$ and $\Lambda=550 \mathrm{~nm}$. For these calculations, the constant grating parameters were kept: ridge height, $h=50 \mathrm{~nm}$; polymer layer thickness, $H=$ $100 \mathrm{~nm}$; polymer refractive index, $n_{\mathrm{d}}=1.5$. The respective results are gathered in Figs. 5 and 6. Upon analyzing them, we have found that the same period of dielectric and metal grating results in obtaining different wavelengths of SPP excitation (cf. Fig. 5). For example, the SRG grating on the dielectric with a period of $\Lambda=400 \mathrm{~nm}$ couples the SPP at a wavelength of $\lambda=675 \mathrm{~nm}$, and a metallic ridge grating of the same period couples SPP at a much longer wavelength of $\lambda=745 \mathrm{~nm}$. For a finite number of ridges, $N=8$, the

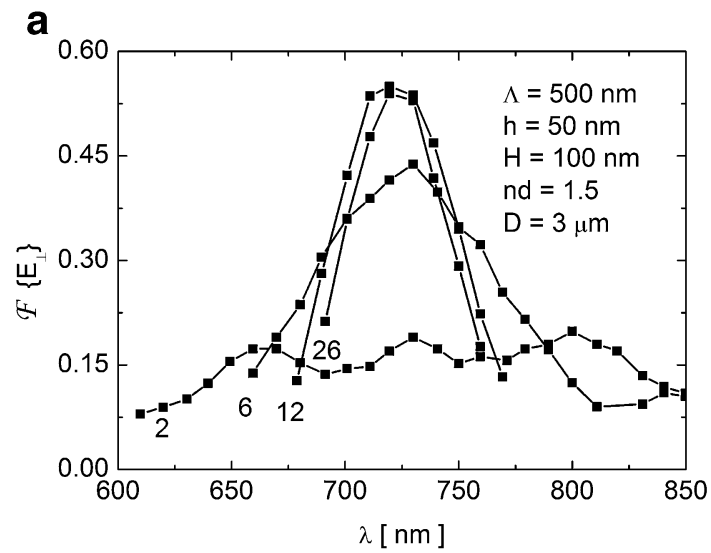

Fig. 7 a Plot of electric field amplitude perpendicular to the metal surface $F\left\{E_{\perp}\right\}$ peak at a constant distance from the grating. SPP was coupled via dielectric grating, Gaussian beam diameter, $D=3 \mu \mathrm{m}$. b

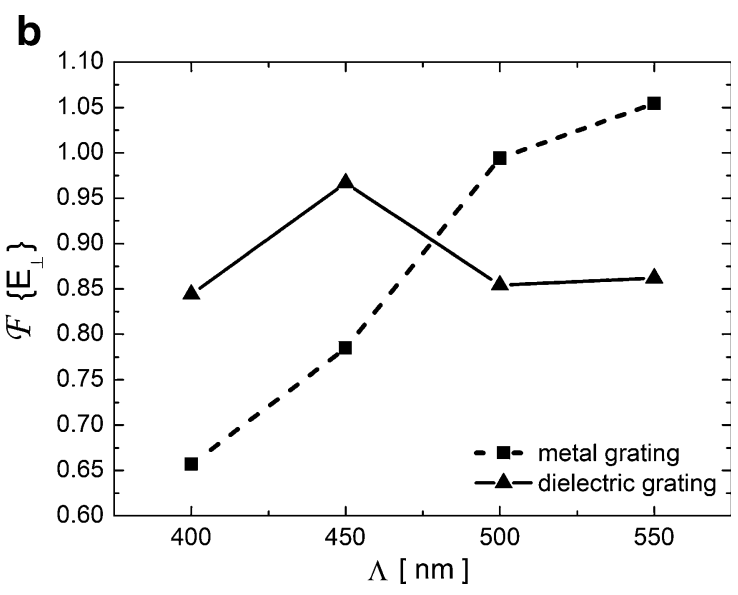

dependence of maximal field amplitude perpendicular to the metal surface $F\left\{E_{\perp}\right\}$ of excited SPP on period of the grating $\Lambda$ (b) for metallic and dielectric gratings

plasmon wavelength almost does not change, and efficiencies of its coupling for dielectric and metal gratings are comparable (cf. Fig. 6). Obviously, an increase of plasmonic band width with respect to that observed for the infinite grating case is observed.

Results are distinctively different when infinite gratings are involved. The coupled SPP wavelengths agree with the trend observed for the finite grating case, but large differences can be observed in their electric field amplitudes (cf. Fig. 5). Dielectric infinite grating couples light into SPPs with ten times lower efficiency than does metal grating (cf. Fig. 5a). However, the electric field measured inside the grating (for the infinite grating this is the only possibility) is composed of the SPP and light propagating and scattered in all possible directions. Also, the infinitely expanded light source can affect the results. Therefore, we conclude that the observation of SPP at some distance after the grating end should give more reliable results. In this

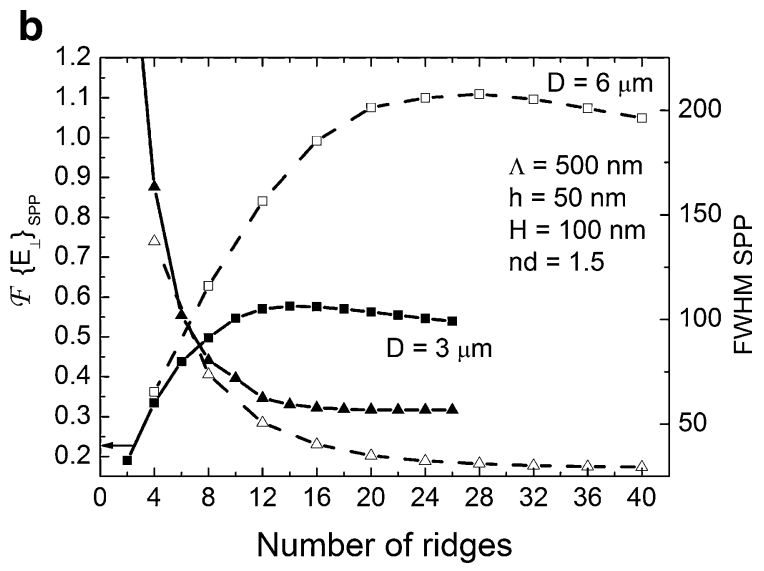

Plot of maximal perpendicular component of electric field $F\left\{E_{\perp}\right\}$ and FWHM of excited SPP band for two Gaussian beam diameters, $D=$ $3 \mu \mathrm{m}$ (solid line) and $D=6 \mu \mathrm{m}$ (dashed line) 
case, the SPP excitation efficiency is similar for metal and polymer gratings - what is shown in Fig. 6a, b.

Furthermore, using the FDTD method, we investigated how the number of ridges influences the coupled SPP peak shape, e.g., its intensity and spectral width. The exemplary SPP bands calculated for the different number of ridges are shown in Fig. 7. One can notice that for $N=6$, an SPP peak is already well characterized. In Fig. 7b, we plotted the SPP peak intensity and the full width at half maximum (FWHM) of the band in function of a number of ridges for excitation with two different Gaussian light beams with diameters, $D=3 \mu \mathrm{m}$ and $D=6 \mu \mathrm{m}$, respectively. It can be seen that the SPP peak intensity reaches the maximum value for a certain number of ridges, $N$, which are related to the diameter $D$ of the light beam. Let us define this number of ridges as the efficient number (EN) of ridges and the cumulative length corresponding to it as the efficient length (EL) of the grating. We suppose that for the EN ridges, the efficiency of SPP coupling and reflection plus decoupling are equal. Any supplementary ridge makes that reflection or/and decoupling of SPP start to dominate. The FWHM decreases with the number of ridges and reaches a minimal value that depends on the excitation beam radius or more precisely on the EN. For the most effective coupling, the EL of grating is larger than the used Gaussian beam diameter. For $D=3 \mu \mathrm{m}$, the beam diameter $\mathrm{EN}=12$ and $\mathrm{EL}=6 \mu \mathrm{m}$; for $D=6 \mu \mathrm{m}, \mathrm{EN}=24$ and $\mathrm{EL}=12 \mu \mathrm{m}$. It can be seen that the EN and EL scale linearly with the Gaussian beam diameter $D$, and EL is about two times larger than $D$.

By the FDTD method, we have shown that the wavelength of the coupled SPP is nearly a linear function of the $n_{\mathrm{d}}$ refractive index of the polymer layer. In the range of 1.45-1.6, the SPP wavelength shifts from $680 \mathrm{~nm}$ to $770 \mathrm{~nm}$ for $\Lambda=500 \mathrm{~nm}$.

In conclusion, we have presented a numerical investigation of the excitation of surface plasmon polaritons using infinite and finite grating inscribed in a polymer layer deposited over a flat gold surface. The geometrical parameters of the gratings have been optimized for the enhanced excitation of SPP showing that a grating amplitude of less than $100 \mathrm{~nm}$ is sufficient for an effective light coupling into plasmon excitation even for a small number of ridges not exceeding ten. The excitation efficiency of SPP for finite dielectric grating is comparable with similar gratings made in metal. Studies of SPP excitation on a different number of ridges show that light beam diameter and number of ridges should carefully be adjusted to achieve efficient SPP excitation.

Acknowledgments This research was supported by the Polish Ministry of Education and Science grant no. N N507 475237 and in part by Wroclaw University of Technology.
Open Access This article is distributed under the terms of the Creative Commons Attribution Noncommercial License which permits any noncommercial use, distribution, and reproduction in any medium, provided the original author(s) and source are credited.

\section{References}

1. Takahara J, Yamagishi S, Taki H, Morimoto A, Kobayashi T (1997) Guiding of a one-dimensional optical beam with nanometer diameter. Opt Lett 22:475-478

2. Barnes WL, Dereux A, Ebbesen TW (2003) Surface plasmon subwavelength optics. Nature 424:824-830

3. Homola J, Yee SS, Gauglitz G (1999) Surface plasmon resonance sensors: review. Sens Actuators B54:3-15

4. Haes AJ, Hall WP, Chang L, Klein WL, Van Duyne RP (2004) A localized surface plasmon resonance biosensor: first steps toward an assay for Alzheimer disease. Nano Lett 4:1029-1034

5. Maier SA, Brongersma ML, Kik PG, Meltzer S, Requicha AAG, Atwater HA (2001) Plasmonics - a route to nanoscale optical devices. Adv Mater 13:1501-1505

6. Nikolajsen T, Leosson K, Bozhevolnyi SI (2004) Surface plasmon polariton based modulators and switches operating at telecom wavelengths. Appl Phys Lett 85(24):5833-5835

7. Zia R, Schuller JA, Brongersma ML (2006) Plasmonics: the next chip-scale technology. Mater Today 9:20-27

8. Maier SA (2007) Plasmonics: fundamentals and applications. Springer Science, New York

9. Ritchie RH, Arakawa ET, Cowan JJ, Hamm RN (1968) Surfaceplasmon resonance effect in grating diffraction. Phys Rev Lett 21:1530-1532

10. Yoon KH, Shuler ML, Kim SJ (2006) Design optimization of nano-grating surface plasmon resonance sensors. Opt Express $14: 4842-4849$

11. Leveque G, Martin OJF (2006) Optimization of finite diffraction gratings for the excitation of surface plasmons. J Appl Phys 100:124301

12. Wang J (2009) Biomolecule-functionalized nanowires: from nanosensors to nanocarriers. Chemphyschem 10:1748-1755

13. Krasavin AV, Zayats AV (2010) All-optical active components for dielectric-loaded plasmonic waveguides. Opt Commun 283:1581-1584

14. Taflove A, Hagness SC (2000) Computational electrodynamics: the finite-difference time-domain method. ARTECH HOUSE, Inc., Norwood

15. Fang Z, Huang S, Lu Y, Pan A, Lin F, Zhu X (2010) Colorchangeable properties of plasmonic waveguides based on Sedoped CdS nanoribbons. Phys Rev B 82:085403-085407

16. Fang Z, Peng Q, Song W, Hao F, Wang J, Nordlander P, Zhu X (2011) Plasmonic focusing in symmetry broken nanocorrals. Nano Lett 11:893-897

17. Fang Z, Lin C, Ma R, Huang S, Zhu X (2010) Planar plasmonic focusing and optical transport using $\mathrm{CdS}$ nanoribbon. ASC Nano 4(1):72-75

18. Vial A, Grimault AS, Demetrio D, Barchiesi D, de la Chapelle ML (2005) Improved analytical fit of gold dispersion: application to the modelling of extinction spectra with a finite-difference timedomain method. Phys Rev B 71:085416b-085422b

19. Palik ED (2007) Handbook of optical constants of solids. Academic, New York

20. Sobolewska A, Miniewicz A (2007) Analysis of the kinetics of diffraction efficiency during the holographic grating recording in azobenzene functionalized polymers. J Phys Chem B 111:1536-1544

21. Radko IP, Nielsen MG, Albrektsen O, Bozhevolnyi SI (2010) Stimulated emission of surface plasmon polaritons by lead-sulphide quantum dots at near-infrared wavelengths. Opt Exp 18:18633-18638 